\title{
Self-Assembled Monolayer- A Nano Surface Modification
}

\author{
Sowmya M. Kumar¹, Parvathi K. Balakrishnan², Chethan Hedge ${ }^{3}$, Shilpa Dandekeri ${ }^{4}$ \\ ${ }^{1}$ Department of Prosthodontics, ABSMIDS, NITTE (Deemed to be University), Mangaluru, Karnataka, India. \\ 2Department of Prosthodontics, ABSMIDS, NITTE (Deemed to be University), Mangaluru, Karnataka, India. \\ ${ }^{3}$ Department of Prosthodontics, ABSMIDS, NITTE (Deemed to be University), Mangaluru, Karnataka, India. \\ ${ }^{4}$ Department of Prosthodontics, ABSMIDS, NITTE (Deemed to be University), Mangaluru, Karnataka, India.
}

\section{ABSTRACT}

\section{BACKGROUND}

Dental implants are made from one or more biomaterials, with titanium and alloys being the most commonly used biomaterials as they exhibit good biocompatibility, mechanical properties and machinability. Osseointegration as defined by Branemark and the closure of soft tissue is the foundation for the success of dental implants. Improving the success rate of dental implants, reducing treatment time, helping with rapid loading and reducing the incidence of peri-implantitis and periimplant mucositis remains an important area of research in the field of oral implantology. Increasing numbers of studies have focused on altering the surface of titanium and titanium alloy in order to increase their biological activity and facilitate osseointegration and soft tissue healing. To achieve these objectives, various conventional methods exist to alter the surface of titanium, but many revolutionary approaches were implemented with the advent in nanotechnology to efficiently modify the surface. However, with the advent of nanotechnology, an innovative technology called Self Assembly is introduced for surface modification. It is done by imprinting the monolayers on the surface of the biomaterials (titanium or its alloys) by a process called self-assembly. Hence, this paper reviews the progress of the application of the self-assembly technique for the surface modification of titanium and its alloys.

\section{KEY WORDS}

Dental implants, Self-assembly, Titanium, Osseointegration
Corresponding Author: Dr. Shilpa Dandekeri, Department of Prosthodontics, A. B. Shetty Memorial Institute of Dental Sciences, NITTE Deemed to be University, Mangaluru, Karnataka, India.

E-mail: drsowmyamk@yahoo.com

DOI: $10.14260 / j e m d s / 2020 / 351$

Financial or Other Competing Interests: None.

How to Cite This Article:

Kumar SM, Balakrishnan PK, Hedge C, et al. Self-assembled monolayer- a nano surface modification. J. Evolution Med. Dent. Sci. 2020;9(20):1608-1612, DOI: $10.14260 /$ jemds/2020/351

Submission 13-03-2020,

Peer Review 25-04-2020,

Acceptance 02-05-2020,

Published 18-05-2020. 


\section{BACKGROUND}

Dental implants provide a reliable, effective and a predictable tooth replacement. It also offers function and aesthetics to both edentulous and partially edentulous patients, which they had with natural dentition. The other benefits of dental implants are recovery of normal masticatory function together with speech, smile and deglutition. ${ }^{1}$

The history of dental implants has two distinct points; the pre-osseointegration period and the post-osseointegration age. During pre-osseointgration era, cobalt-chromiummolybdenum and different stainless steels were used to develop the blade and plate form implants. However, the success was very limited. Second era or the new paradigm of therapy was opened when the studies on osseointegration of implants were presented to the scientific world by the people like Branemark, Albrektsson $\mathrm{T}$ etc. The biological phenomenon involving direct bone-to-titanium surface contact is the basis of Osseointegration. ${ }^{2}$ It was initially described as "a structural and functional interface between an ordered, living bone and an implant surface."3,4,5 Later the researchers gave it a more comprehensive clinical definition and technically it is "a mechanism in which alloplastic material is clinically asymptomatically accomplished and preserved in the bone during functional charging." 6,7

If the dental implant success rate is improved, the treatment time can be shortened considerably, periimplantitis and peri-implant mucositis can be minimized, thus the osseointegration is an area where researchers are spending some quality time. ${ }^{8}$ From the literature, the main process that defines the success and failure of implant osseointegration occurs largely at the tissue implant interface. Factors like surface- texture, topography, roughness and energy of implant material determine the mechanical stability of the implant tissue interface. It is evident from these observations that the surface of the interface in osseointegration is very critical. Thus, the greatest potential for enhancing the implant dentistry lies on the implant surfaces. ${ }^{9}$

The research to increase the biological activity on the surface of Titanium and Titanium alloy for promoting better osseointegration and soft tissue healing is gaining ground and an umpteen number of researches are being devoted to it. 8 There are many conventional methods to alter the surface of titanium, but many revolutionary approaches were implemented with the advent in nanotechnology to efficiently modify the surface.

Usually most of the conventional surface modification techniques are a top down process, which required multiple interventions and special guidance during the process. With the advent of nanotechnology, an innovative technology called Self Assembly is introduced for surface modification. It is done by imprinting the monolayers on the surface of the biomaterials (titanium or its alloys) by a process called selfassembly. Self-assembly is a bottom up process which required neither the guidance nor the intervention during the assembly process.

This article reviews the present self-assembly method and its application in the dental implants.

\section{Self-Assembly (SA)}

"SA is a type of process in which a disordered system with pre-existing components forms an ordered structure or sequence as a result of specific local interactions among its components without external influence. This mechanism is dependent on weak covalent bonds including hydrogen and ionic bonds or Van der Waals and hydrophobic interactions and can be extended to the manufacture of various complex micro- and nanostructures such as flowers, tubes, micelles, films, mesophase particles and hollow sphere structures."10

SAM is an interface between materials with different chemical and physical property. This changed the focus of surface science, which was early dominated by metal and metal oxides into organic molecules, which turned the surface study into solvents and their contact with biologically relevant surfaces. In practical applications such as chemical sensing, adhesion and implants, it provided an alternative truly innovative implementation of chemical surface treatments. ${ }^{11-21}$

\section{Need for SAM in Dental implant}

As discussed earlier, the implant therapy's focus on osseointegration changed the total outcome of the treatment. The treatment's clinical part is multidisciplinary and better outcome can be predicted if the treatment is planned and controlled well. Even though this phase is well managed, many cases reported failure and the studies showed the evidence for the importance of implant design while planning the treatment. The main aspect of the implant design factor is its surface character.22-28 From early investigation, it was clear that surface property of $\mathrm{Cp}$-Titanium is more biocompatible in terms of surface characteristics. ${ }^{29,30}$ This early knowledge led to the modification of Ti first in micro scale level and later in nano scale; after the advent of nanotechnology.

The application of nanotechnology to alter Ti dental implants surface topography led to the discovery of novel physiochemical behaviours or biochemical events such as bone bonding and cell adhesion. ${ }^{22}$ With self-assembly, the formed monolayer is osteo inductive or a cell adhesive molecule. ${ }^{31}$ To obtain better implants, it was necessary to find a better way to bring changes to the surface topography on the nano scale. Many methods were invented and among them the best is the self-assembly of monolayer on the Ti surface.

\section{Formation of Monolayer by SA}

SAM is a hierarchically organized amphiphilic molecular layer at the end a head group and on the other end or tail a functional group. The presence of end functional group improves the hydrophilic and hydrophobic property of the substrate. SAM process includes bulk solution transfer, specimen surface preparation, surface uptake and twodimensional substrate organization. ${ }^{32}$ Chemisorption of hydrophilic head groups onto the substrate is accompanied by hydrophobic tail group organization in two dimensions to the substrate. Standing up phase will be reached once the alkyl groups are stacked to a striped phase. Last phase is the rearrangements of in which the monolayer will grow fully on the surface until no area is left on the surface.11 
The speed of SAM formation depends on preparation, conditioning of materials and substrate property. Both the stability and packing order of SAM depends on the head group. Chain length of the monolayer is the responsible factor for the monolayer thickness. External considerations, such as surface cleanliness, surfactant concentration, substrate preparation and the purity of the solvent all can affect the monolayer formation on the surface of the substrate $33-35$. Since it is proved that the implants of $\mathrm{Ti}$ with modified surface topography shows the better quality, it is important to discuss the formation of SAM on Ti.

\section{Formation of SAM on Ti}

A pre-treatment should be performed to obtain a clean and smooth substrate for the best deposition of the SAM Ti layer. Contaminants are removed from the surface by using a solvent such as acetone, methanol or ethanol ${ }^{11}$. Mechanical polishing of the surface is done to get a smooth and flat surface. A porous surface is highly undesirable; thus, these chemical pre-treatments should be carefully conducted in order to control the surface modification ${ }^{36}$. Purity of the absorbent or the surfactant is very important for SA. Minute contamination can lead to disordered, non-ideal monolayer. ${ }^{11}$ Silane/Siloxane and phosphonic acid/ phosphonates are the organic reagents used in the SA of Ti monolayer.

Sample is placed in vertical position for the chemical pretreatment. The vertical positioning will remove the microscopically small particles from the surface. The samples were taken out of the chemical solvent before the assembly process and rinsed with high purity water followed by additional washing in the nitrogen stream and UV ozone cleaner. Phosphonic acid was sprayed on the hydroxylated titanium substrate and immediately the cleansed $\mathrm{Ti}$ is immersed in tetrahydrofuran solution. Then the deposition of monolayer corresponding to the phosphate begins on the Ti surface and the entire process is completed in 48 hours. The Ti with phosphate SAM is taken out and rinsed in the pure water and then used for the implant production. ${ }^{11}$

SAM's formation is a potential and economical method for obtaining better osteointegration. SAM is a physiochemical thin film able to modify the chemical characteristics of $\mathrm{Ti}$ surface. Nano level surface integration has great influence in healing process. ${ }^{11}$ On nano level things act very differently and it is the reason for the success of SAM in the implant application.

\section{Nanotechnology of Surface Science}

On nano scale the surface property of the material is controlled by quantum physics. The surface quality of implants depends on its mechanical property, topographic property and physiochemical properties. All these three properties are dependent on each other. Thus, a change in any of these properties results in different outcomes, this is because the quantum physics governs the behaviour in nanoworld. However; this approach conserves the surface chemistry of different topographies and enhance the cell adhesion on the biomaterials. On nanoscale the natural cellular environment can be emulated. It favours rapid healing of the wounds.
Nanotopography alters the cell interaction when compared to conventional surface topography. When implant comes in contact with body its cell integration and proliferation depend on the protein absorption. Cell binding to bio-nano materials are exceptional. With nano material, the protein interaction at the biological site can be altered. When compared to conventional materials, the nano-scale surface modified implants increase the vitronectin absorption. From the studies it is evident that the fibronectin adhesion on hydrophilic SAM is more than what is anticipated. It is considered as the proof for the osteoblast adhesion. ${ }^{37-42}$ Other than these SAM is showing great selective adhesion of cells, proteins and increased osteoblast proliferation. ${ }^{43,44}$ Bacterial adhesion is minimal in SAM. ${ }^{45}$ Thus it is obvious that the nano-scale alteration of surface topography using SAM is revolutionary and improving the quality of implant treatment.

\section{APPLICATION OF NANO-SCALE} MODIFIED TI IMPLANTS

\section{TI Implant with Better Biologically Active Surface for Enhanced Osseointegration}

The osseointegration of bone with dental materials is improved with bioactive surfaces. The implant's surface roughness affects bone to implant contact and the implant's primary stability. ${ }^{2}$ Way the cells respond to smooth, rough surfaces may change the process of osseointegration. The surface property of the implant therefore plays an important role in the biological interaction between the implant and the host bone. At nano scale, surface energy increases, which in turn increases surface wettability to the blood, cell adhesion to the surface and induces fibrin binding, matrix proteins, growth and differentiation factors. It can also influence the process of cell migration, proliferation and differentiation, thereby enhancing the step of osseointegration by promoting wound healing after implantation. ${ }^{46}$

TI Implant with Antibacterial and Antimicrobial Property There are possibilities for inflammation on the implant site as the bacteria colonize and grow biofilms on the osseointegrated dental implant transmucosal abutment. In SAM, a lot of work is taking place around the world to coat the implant with a thin film of antimicrobial substances as well as to confine the drugs on the surfaces of the implants for release into the implant bed to prevent bacterial adhesion. In other words, the SAM helps practitioners to combat inflammation by immobilizing biomolecules like antibodies, antibiotics and antimicrobial peptides by binding affinity to the implant surface ${ }^{2}$. Bisphosphate loaded implants and simvastatin trapped implants are already being tested in the research labs. More advancement is required in the field for more effectiveness of this treatment methodology ${ }^{46}$.

\section{LIMITATION OF THE SAM SURFACE MODIFICATION IMPLANTS}

It is not exactly the limitation, but the reproducibility of an ideal surface is still a question for the researchers. With chemical treatment, reproducible surface roughness in the 
nanometer is difficult to obtain. Although all modified surfaces that display nano-topography, most of them will not have significant nanostructures that enable cell migration, attachment, proliferation, and differentiation. ${ }^{46}$

\section{CONCLUSIONS}

Titanium and its alloys are the first choice as implant materials for most practitioners, owing to their excellent biocompatibility and mechanical properties. Surface energy, surface composition, surface roughness and surface topography may affect the treatment outcome significantly. Thus, the surface modification of the implant is very important for obtaining better treatment outcome. With the help of nanotechnology, it is possible to perform ideal surface modification on the implant surfaces. SAM is one of the best technologies to do a surface modification on the implants at nano-level. These modified surfaces give better results than the implants with micro-scale surface modification, especially in osseointegration. In addition to this, SAM also opens the door for targeted and localized drug delivery to control the post implant inflammation. It also helps to fight the bacterial and microbial infection as SAM gives the provision to design the surface with antimicrobial monolayer coatings.

Though the SAM surface modification technique on dental implants is a promising breakthrough in the implantology, it is not yet commercialized as the reproducibility of the ideal implant surface is not yet achieved. However, it is certain that one day, the laboratory researchers will help the clinicians to achieve the successful implant treatment with SAM that promotes ideal healing process without compromising the cost and quality.

\section{REFERENCES}

[1] Tagliareni JM, Clarkson E. Basic concepts and techniques of dental implants. Dent Clin North Am 2015;59(2):25564.

[2] Freitas SC, Correa-Uribe A, Martins MC, et al. Selfassembled monolayers for dental implants. Int Dent J 2018;2018:4395460.

[3] Sykaras N, Iacopino AM, Marker VA, et al. Implant materials, designs and surface topographies: their effect on osseointegration. A literature review. Int J Oral Maxillofac Implants 2000;15(5):675-90.

[4] Zarb GA. Introduction to osseointegration in clinical dentistry. J Prosthet Dent 1983;49(6):824.

[5] Zarb GA, Albrektsson T. Osseointegration: a requiem for the periodontal ligament? Int J Periodontics Restorative Dent 1991;11(1):88-91.

[6] Albrektsson T, Zarb G, Worthington P, et al. The longterm efficacy of currently used dental implants: a review and proposed criteria of success. Int J Oral Maxillofac Implants 1986;1(1):11-25.

[7] Zarb GA, Albrektsson T. Towards optimized treatment outcomes for dental implants. J Prosthet Dent 1998;80(6):639-40.
[8] Shi Q, Qian Z, Liu D, et al. Surface modification of dental titanium implant by layer-by-layer electrostatic selfassembly. Frontiers in Physiology 2017;8:574.

[9] Parekh RB, Shetty O, Tabassum R. Surface modifications for endosseous dental implants. The International Journal of Oral Implantology and Clinical Research 2012;3(3):116-21.

[10] Kajbafvala A, Bahmanpour H, Maneshian MH, et al. Selfassembly techniques for nanofabrication. J Nanomater 2013;2013:158517.

[11] Mastrangelo F, Fioravanti G, Quaresima R, et al. Selfassembled monolayers (SAMs): which perspectives in implant dentistry? J Biomater \& Nanobiotechnol 2011;2(5):533-43.

[12] Raman A, Quiñones R, Barriger L, et al. Understanding organic film behavior on alloy and metal oxides. Langmuir 2010;26(3):1747-54.

[13] Rozsnyai LF, Wrighton MS. Controlling the adhesion of conducting polymer films with patterned self-assembled monolayers. Chem Mater 1996;8(2):309-11.

[14] Nozawa K, Nishihara $H$, Aramaki K. Chemical modification of alkanethiol monolayers for protecting iron against corrosion. Corros Sci 1997;39(9):1625-39.

[15] Svedhem S, Hollander CÅ, Shi J, et al. Synthesis of a series of oligo (ethylene glycol)-terminated alkanethiol amides designed to address structure and stability of biosensing interfaces. J Org Chem 2001;66(13):4494-503.

[16] Kovaric BC, Kokona B, Schwab AD, et al. Self-assembly of peptide porphyrin complexes: toward the development of smart biomaterials. J Am Chem Soc 2006;128(13):4166-7.

[17] Wang DA, Feng LX, Ji J, et al. Novel human endothelial cell-engineered polyurethane biomaterials for cardiovascular biomedical applications. J Biomed Mater Res A 2003;65(4):498-510.

[18] Jiang Y, Wang Z, Yu X, et al. Self-assembled monolayers of dendron thiols for electrodeposition of gold nanostructures: toward fabrication of superhydrophobic/superhydrophilic surfaces and $\mathrm{pH}$ responsive surfaces. Langmuir 2005;21(5):1986-90.

[19] Park BS, Heo SJ, Kim CS, et al. Effects of adhesion molecules on the behavior of osteoblast-like cells and normal human fibroblasts on different titanium surfaces. J Biomed Mater Res A 2005;74(4):640-51.

[20] Fan FR, Yao Y, Cai L, et al. Structure-dependent charge transport and storage in self-assembled monolayers of compounds of interest in molecular electronics: effects of tip material, headgroup and surface concentration. J Am Chem Soc 2004;126(12):4035-42.

[21] Nitahara S, Akiyama T, Inoue S, et al. A photoelectronic switching device using a mixed self-assembled monolayer. J Phys Chem B 2005;109(9):3944-8.

[22] Mendonça G, Mendonça DBS, Aragao FJL, et al. Advancing dental implant surface technology-from micron-to nanotopography. Biomaterials 2008;29(28):3822-35.

[23] Adell R, Eriksson B, Lekholm U, et al. A long-term followup study of osseointegrated implants in the treatment of totally edentulous jaws. Int J Oral Maxillofac Implants 1990;5(4):347-59.

[24] Adell R, Lekholm U, Rockler B. et al. A 15-year study of osseointegrated implants in the treatment of the edentulous jaw. Int J Oral Surg 1981;10(6):387-416. 
[25] Zarb GA, Schmitt A. The longitudinal clinical effectiveness of osseointegrated dental implants: the Toronto study. Part III: problems and complications encountered. J Prosthet Dent 1990;64(2):185-94.

[26] Morton D, Jaffin R, Weber HP. Immediate restoration and loading of dental implants: clinical considerations and protocols. Int J Oral Maxillofac Implants 2004;(Suppl 19)103-8.

[27] Cooper LF. Biologic determinants of bone formation for osseointegration: clues for future clinical improvements. J Prosthet Dent 1998;80(4):439-49.

[28] Stanford CM, Johnson JK, Fakhry A, et al. Outcomes of a fluoride modified implant one year after loading in the posterior-maxilla when placed with the osteotome surgical technique. Appl Osseointegration Res 2006;5:50-5.

[29] Kasemo B. Biocompatibility of titanium implants: surface science aspects. J Prosthet Dent 1983;49(6):832-7.

[30] Johansson CB, Albrektsson T. A removal torque and histomorphometric study of commercially pure niobium and titanium implants in rabbit bone. Clin Oral Implants Res 1991;2(1):24-9.

[31] Scotchford CA, Gilmore CP, Cooper E, et al. Protein adsorption and human osteoblast-like cell attachment and growth on alkylthiol on gold self-assembled monolayers. J Biomed Mater Res 2002;59(1):84-99.

[32] Schreiber F. Structure and growth of self-assembling monolayers. Prog Surf Sci 2000;65(5-8):151-256.

[33] Webster TJ, Siegel RW, Bizios R. Osteoblast adhesion on nanophase ceramics. Biomaterials 1999;20(13):1221-7.

[34] Webster TJ, Ergun C, Doremus RH, et al. Enhanced functions of osteoblasts on nanophase ceramics. Biomaterials 2000;;21(17):1803-10.

[35] Schwartz Z, Nasazky E, Boyan BD. Surface microtopography regulates osteointegration: the role of implant surface microtopography in osteointegration. Alpha Omegan 2005;98(2):9-19.

[36] Ajami E, Aguey-Zinsou KF. Formation of OTS selfassembled monolayers at chemically treated titanium surfaces. J Mater Sci Mater Med 2011;22(8):1813-24.
[37] Brody S, Anilkumar T, Liliensiek S, et al. Characterizing nanoscale topography of the aortic heart valve basement membrane for tissue engineering heart valve scaffold design. Tissue Eng 2006;12(2):413-21.

[38] Hansen JC, Lim JY, Xu LC, et al. Effect of surface nanoscale topography on elastic modulus of individual osteoblastic cells as determined by atomic force microscopy. J Biomech 2007;40(13):2865-71.

[39] Wu SJ, De Jonghe LC, Rahaman MN. Sintering of Nanophase $\gamma-\mathrm{Al}_{2} \mathrm{O}_{3}$ powder. $\mathrm{J}$ Am Ceram Soc 1996;79(8):2207-11.

[40] Baraton MI, Chen X, Gonsalves KE. FTIR study of a nanostructured aluminum nitride powder surface: Determination of the acidic/basic sites by CO, CO2 and acetic acid adsorptions. Nanostructured Materials 1997;8(4):435-45.

[41] Sinha RK, Tuan RS. Regulation of human osteoblast integrin expression by orthopedic implant materials. Bone 1996;18(5):451-7.

[42] Arima Y, Iwata H. Effect of wettability and surface functional groups on protein adsorption and cell adhesion using well-defined mixed self-assembled monolayers. Biomaterials 2007;28(20):3074-82.

[43] Price RL, Gutwein LG, Kaledin L, et al. Osteoblast function on nanophase alumina materials: Influence of chemistry, phase and topography. J Biomed Mater Res A 2003;67(4):1284-93.

[44] McManus AJ, Doremus RH, Siegel RW, et al. Evaluation of cytocompatibility and bending modulus of nanoceramic/polymer composites. J Biomed Mater Res A 2005;72(1):98-106.

[45] Colon G, Ward BC, Webster TJ. Increased osteoblast and decreased Staphylococcus epidermidis functions on nanophase $\mathrm{ZnO}$ and TiO2. J Biomed Mater Res A 2006;78(3):595-604.

[46] Anil S, Anand PS, Alghamdi H, et al. Dental implant surface enhancement and osseointegration. Implant Dentistry-A Rapidly Evolving Practice 2011;29:83-108. 